\section{Quantifizierter Gleason-Score verbessert prognostische Aussagekraft}

\author{
Deutsche Mediziner haben eine Modifikation des Gleason-Scores vorgeschla- \\ gen, die dessen prognostischen Wert noch steigern könnte.
}

$\mathrm{D}$ e Aggressivität von Prostatakarzinomen mithilfe des Gleason-Gradings und des Gleason-Scores zu beurteilen, ist längst Standard. Ein Forscherteam um Guido Sauter vom Universitätsklinikum Hamburg-Eppendorf betrachtet die Aggressivität von Prostatakarzinomen als kontinuierliche Größe, die den Rahmen kategorialer Einteilungen sprengt. Sie wollen das Gleason-System deshalb ergänzen.

Sauter und Kollegen schlagen vor, beim Gleason-Score nicht nur die Summe und deren Summanden, sondern auch den prozentualen Anteil am Tumorgewebe in Stanze oder Prostatektomie anzugeben, den die einzelnen Grade einnehmen. Auf diese Weise entstünde eine Art quantifiziertes GleasonMuster. Die Auswirkungen auf die Prognose im Sinne eines biochemischen Rezidivs lassen sich damit immer feiner abschätzen. Wichtig könnte das vor allem für den Gleason-Score $3+4$ sein. Hier ist die Breite der therapeutischen Möglichkeiten am größten. Ergebnisse von Studien lassen vermuten, dass zumindest manche dieser Tumoren für eine aktive Überwachung geeignet sein könnten.

Sauter et al. teilten die Kategorie $3+4$ weiter ein, etwa danach, ob der Anteil von Gleason-Score 4 bei 1-24\% oder bei 25-49 \% lag. Die biochemischen Rückfallraten über einen Zeitraum von sechs Jahren erreichten bei niedrigem Score4-Anteil rund $15 \%$, bei hohem Anteil etwa $35 \%$. Insgesamt war die Rückfallquote umso höher, je mehr Score-4-Gewebe die Proben aufwiesen. Ab einem Score-4-Anteil von mehr als $80 \%$ verhalten sich Tumoren mit Gleason-Score $4+3$ sehr ähnlich wie Karzinome mit Gleason-Score 8.

Fazit: ,Mit Blick auf die Unsicherheit, ob $3+4$-Karzinome aktiv überwacht werden sollten oder nicht, ist man versucht zu spekulieren, dass es sich bei Karzino- men mit niedrigem Grad-4-Anteil um jene aus dieser Gruppe handelt, die sich für die Überwachungsstrategie eignen“, schreiben die Hamburger Forscher. Bei höheren Anteilen sei wohl eine aggressivere Therapie angezeigt. Mit ihren Daten zum Score-4-Anteil in Prostatabiopsien sehen sie dieses Konzept stark unterstützt. Die quantifizierende Angabe mache laut Sauter et al. das Ergebnis auch unabhängiger von der Variabilität der pathologischen Beurteilung je nach Untersucher.

Dr. Robert Bublak

Sauter $\mathrm{G}$ et al. Clinical utility of quantitative gleason grading in prostate biopsies and prostatectomy specimens. Eur Urol. 2016;69(4):592-8.

\section{Kommentar}

In ihrer Studie mit 12.150 Patienten, die zwischen 2005 und 2014 an der Martiniklinik radikal prostatektomiert wurden, wiesen Sauter et al. nach, dass die traditionelle Gleason-Klassifikation wie aus der Literatur bekannt, eine klare prognostische Aussagekraft zeigt - mit zunehmend schlechterem Outcome für die Gleason-Scores $3+3=6,3$ $+4=7,4+3=7,8$, und 9-10. Darüber hinaus konnten die Forscher aber auch zeigen, dass die weiterführende Quantifizierung der Grade $3+4=7$ und $4+3=7$ mittels prozentualer Differenzierung in die Gruppen $3+4$ $=7$ "low" $(1-24 \%$ Gleason 4$)$ versus $3+4=7$ "high" (25-49\% Gleason 4) und 4 + 3= 7 "low" (50-74\% Gleason 4) versus 4 + $3=7$ "high" (75-94\% Gleason 4) die Outcomes noch akkurater vorhersagen lässt.

Für die Subklassifikation der Low-RiskKarzinome wies die Arbeitsgruppe um Sauter nach, dass sich Karzinome mit Gleason-Score $3+4$ bei geringem Anteil des Gleason-Scores 4 (1-24\%) sehr ähnlich wie Karzinome mit Gleason-Score 3 + $3=6$ entwickeln. Bei den High-Risk-Karzinomen andererseits, verhält sich ein Tumor mit Gleason-Score $3+4$ und hohem Anteil des Gleason-Scores 4 (75-94\%) prognostisch ähnlich schlecht wie ein Karzinom mit Gleason-Score $4+4=8$.
Die Autoren dieser retrospektiven Untersuchung plädieren für eine differenziertere Gleason-Score-Analyse radikaler Prostatektomiepräparate: Sie propagieren die differenziertere Quantifizierung der einzelnen Gleason-Muster, um klinische Outcomes präziser vorhersagen zu können. Sie ermögliche, besser zu selektieren, welche Patienten einer Active Surveillance (auch Gleason $3+4$ mit wenig Gleason 4 Anteilen) oder einer aggressiveren Therapie (z. B. Gleason-Score $3+4$ mit hohem Gleason-Score 4 Anteil) zugeführt werden können.

Fazit: Den Autoren dieser Studie muss zunächst für ihre Arbeit gratuliert werden, weil sie einmal mehr an den in der Martiniklinik generierten sehr hohen Fallzahlen hochinteressante klinische Zusammenhänge zwischen pathologischem Befund und Outcome darstellen können. Es ist jedoch anzumerken, dass es sich ausschließlich um Prostatektomiepräparate handelte, sodass die Schlussfolgerung, auch verbesserte Aussagen zur Active Surveillance treffen zu können, mit Vorsicht beurteilt werden muss. Eine ähnliche Studie an Prostatastanzbiopsien kombiniert mit einer prospektiven Outcome-Analyse wäre wünschenswert. Doch genau hier liegt eines der Probleme in der aktuellen Versorgungssituation: So schön die Ergebnisse aus Hamburg sind, es wird schwierig sein, diese Daten eines hochspezialisierten Zentrums auf ganz Deutschland zu übertragen. Noch immer sind die pathologischen Aufarbeitungen von Prostatabiopsien, aber auch Prostatektomiepräparaten nicht standardisiert genug, um von einer Gültigkeit für alle Prostatakarzinompatienten auszugehen. Zudem gibt es zu wenig prospektive OutcomeRegister, die eine prospektive Validierung dieser Daten für alle Prostatakarzinombehandler und -patienten zuließe. Hier gibt es noch viel zu tun.

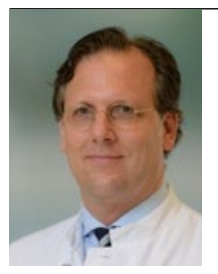

Prof. Dr. med. Christian Wülfing

Abteilung für Urologie Asklepios Klinik Altona Paul-Ehrlich-Str. 1 22763 Hamburg E-Mail: c.wuelfing@asklepios.com 